\title{
Pancreatitis aguda por hipertrigliceridemia. Uso de plasmaféresis: reporte de caso
}

\author{
Acute pancreatitis due to hypertriglyceridemia. Use of plasmapheresis: case \\ report
}

Pancreatite aguda devido à hipertrigliceridemia. Uso da plasmapheresis: relato de caso

\author{
Danny Fernando Silva Cevallos (D) a, Mirella Barrera Rivera (D) ${ }^{b}$, Diana Stefania Evangelista \\ Barragán (iD ${ }^{\text {b }}$, César Enrique Arreaga Pérez (DD
}

\begin{abstract}
a Servicio de Emergencias y Hospitalización, Clínica Guayaquil, Guayaquil-Ecuador; ' Servicio de Medicina Interna, Clínica Guayaquil, Guayquil-Ecuador.
\end{abstract}

Correspondencia a:

Danny Fernando Silva Cevallos, dannyskel40@gmail.com

Recibido: 15 de octubre, 2020 Aceptado: 19 de noviembre, 2020 Publicado: 4 de enero, 2021

CASO CLÍNICO

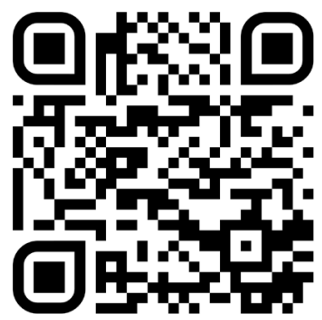

Escanea en tu dispositivo móvil o revisa este artículo en: https:// revistaclinicaguayaquil.org

\section{RESUMEN}

La pancreatitis aguda por hipertrigliceridemia (PATG) se describe como una causa poco común de pancreatitis, con una incidencia que oscila entre el 2 al 4\% de los casos; genera una morbilidad y mortalidad importante de aproximadamente 40 por cada 100.000 personas. Varios son los mecanismos fisiopatológicos que explican la aparición de pancreatitis causada por hipertrigliceridemia; uno de ellos es el papel tóxico directo de los ácidos grasos libres sobre el tejido pancreático $y$, otro mecanismo ampliamente acogido, es la obstrucción vascular mediada por los quilomicrones, dada la disminución de la expresión genética de la lipoproteína lipasa. La American Society for Apheresis (ASFA) indica el uso de plasmaféresis en casos de pancreatitis severa, cuando los niveles de triglicéridos exceden los $2000 \mathrm{mg} / \mathrm{dl}$ y cuando no hay respuesta satisfactoria al tratamiento de primera línea. El objetivo del uso de plasmaféresis y éxito del tratamiento se basa en un descenso de los triglicéridos por debajo de $500 \mathrm{mg} / \mathrm{dl}$. En este caso se demostró que la valoración oportuna de la paciente y la instauración del tratamiento con plasmaféresis en el tiempo adecuado fue fundamental para la resolución de su patología.

Palabras clave: pancreatitis aguda; hipertrigliceridemia; plasmaféresis; triglicéridos
ABSTRACT
Acute hypertriglyceridemic pancreatitis (HTGP) is described as an uncommon cause of pancreatitis, with an incidence ranging from $2 \%$ to $4 \%$ of cases; it generates significant morbidity and mortality of approximately 40 per 100,000 inhabitants. There are several pathophysiological mechanisms that explain the appearance of pancreatitis caused by hypertriglyceridemia; one of them is the direct toxic effect of free fatty acids on pancreatic tissue, and another widely accepted mechanism is chylomicron-mediated vascular obstruction, given the decrease in the genetic expression of lipoprotein lipase. The American Society of Apheresis (ASFA) indicates the use of plasmapheresis in cases of severe pancreatitis when triglyceride levels exceed $2000 \mathrm{mg} / \mathrm{dl}$ and when there is no satisfactory response to first- line treatment. The goal of the use of plasmapheresis and treatment success is based on a decrease in triglyceride levels below $500 \mathrm{mg} / \mathrm{dl}$. In this case report, it was demonstrated that the timely assessment of the patient and the establishment of treatment with plasmapheresis in the appropriate time was essential for the resolution of her pathology. 
Key words: acute pancreatitis; hypertriglyceridemia; plasmapheresis; triglycerides

\section{RESUMO}

A pancreatite hipertrigliceridêmica aguda (PATG) é descrita como causa rara de pancreatite, com incidência variando de $2 \%$ a $4 \%$ dos casos; gera morbidade e mortalidade significativas de aproximadamente 40 por 100.000 pessoas. Vários são os mecanismos fisiopatológicos que explicam o aparecimento da pancreatite causada pela hipertrigliceridemia; um deles é o papel tóxico direto dos ácidos graxos livres no tecido pancreático, e outro mecanismo amplamente aceito é a obstrução vascular mediada por quilomícrons, dada a diminuição da expressão genética da lipoproteína lipase. $A$ American Society of Apheresis (ASFA) indica o uso da plasmaférese nos casos de pancreatite grave, quando os níveis de triglicerídeos ultrapassam $2.000 \mathrm{mg} / \mathrm{dl}$ e quando não há resposta satisfatória ao tratamento de primeira linha. 0 objetivo do uso da plasmaférese e o sucesso do tratamento é baseado na diminuição dos triglicerídeos abaixo de 500 mg / dl. Nesse caso, ficou demonstrado que a avaliação oportuna da paciente e o estabelecimento do tratamento com plasmaférese em tempo adequado foram essenciais para a resolução de sua patologia.

Palavras-chave: pancreatite aguda; hipertrigliceridemia; plasmaferese; triglicerídeos

\section{INTRODUCCIÓN}

Se describe a la pancreatitis como un proceso inflamatorio que afecta a la glándula; entre las causas más comunes se encuentran las inducidas por cálculos biliares y el abuso de alcohol, dejando a la pancreatitis por hipertrigliceridemia como una causa poco común con una incidencia que oscila entre el $2 \%$ al $4 \%$ de los casos y genera un morbimortalidad de 40 por cada 100.000 personas $(1,2)$. Se han realizado estudios para establecer el corte de triglicéridos que pueden provocar pancreatitis aguda, aunque se desconoce aún exactamente, se han establecido niveles mayores a 1000 mg/ dl (3).

Aunque la fisiopatología no está claramente establecida, se sabe que es el resultado de interacciones entre múltiples factores, siendo la teoría más aceptada sobre el metabolismo excesivo por la lipasa pancreática que produce la hidrólisis de los triglicéridos y genera como consecuencia la liberación de ácidos grasos libres, junto con la hiperviscosidad producida por el exceso de quilomicrones en los capilares pancreáticos, lo que puede provocar isquemia (4-6). Además, se asocia a trastornos genéticos como la hipertrigliceridemia familiar por la elevación de la apolipoproteína B y del colesterol no HDL (3).

Existen complicaciones locales como necrosis, abscesos, pseudoquiste pancreático y ascitis; así mismo, se pueden presentar complicaciones sistémicas que comprenden desde hemorragia digestiva, hiperglicemia, hipercalcemia, cetoacidosis diabética y síndrome de dificultad respiratoria aguda, hasta la disfunción multiorgánica, coagulación intravascular diseminada y síndrome compartimental (7).

La plasmaféresis es el método en el que se disgrega el plasma de la sangre para realizar su depuración y posteriormente infundir el plasma depurado al paciente (8). Se utiliza como opción de tratamiento en PATG, ya que elimina rápidamente los triglicéridos y quilomicrones de la circulación, deteniendo así, el factor que desencadena el cuadro clínico y su inflamación característica. En comparación con el tratamiento conservador, este tratamiento se ha propuesto especialmente en pacientes críticos debido a la rapidez su efecto (8).

Actualmente, las guías determinan terapias ante esta patología cuya finalidad es tratar la hipertrigliceridemia; dentro de las opciones disponibles se encuentran los hipolipemiantes orales, insulina, heparina y plasmaféresis $(4,8)$. La Sociedad Americana de Aféresis (American Society for Apheresis, ASFA) indica el uso de plasmaféresis en aquellos pacientes con pancreatitis severa cuando los niveles de triglicéridos se exceden los 2000 mg/dl y cuando no hay respuesta satisfactoria al tratamiento de primera línea; se ha evidenciado entre el 46 al $80 \%$ de reducción de síntomas con 2 a 3 sesiones de plasmaféresis (8-10). El objetivo del uso de plasmaféresis y éxito del tratamiento se basa en un descenso de los triglicéridos por debajo de $500 \mathrm{mg} / \mathrm{dl}$ (7). Existen contraindicaciones para el uso de plasmaféresis como infarto agudo de miocardio con inestabilidad hemodinámica, hemorragia intracraneal o edema cerebral severo y más de 72 horas desde el inicio de la PATG.

\section{DESCRIPCIÓN DEL CASO CLÍNICO}

Paciente femenino de 32 años con antecedentes de diabetes mellitus tipo 2, tratada con glimepirida/metformina 2/850 mg/día, hipertensión arterial diagnostica hace 2 años, no controlada. La paciente ingresa con un cuadro clínico de aproximadamente 8 horas de 
evolución, que se caracteriza por dolor abdominal en hipocondrio izquierdo con irradiación dorsal en hemicinturón, de gran intensidad $8 / 10$, el cual no cede con analgesia y se acompaña de náuseas y vómitos en 3 ocasiones de contenido alimenticio, precedida de la ingesta excesiva de comida copiosa y ricas en grasas. Al examen físico las mucosas se encontraban secas, el abdomen blando y depresible, doloroso a la palpación profunda, dolor en hemicinturón, ruidos hidroaéreos aumentados. Se coloca una sonda nasogástrica, por donde se evidencia débito bilioso. Hemodinámicamente estable con signos vitales: presión arterial $134 / 74 \mathrm{mmHg}$, frecuencia cardiaca de $89 \mathrm{lpm}$, frecuencia respiratoria de 19 rpm, saturación periférica de oxígeno $98 \%$ aire ambiente, glicemia $333 \mathrm{mg} / \mathrm{dl}$. De acuerdo al cuadro clínico y exámenes complementarios (Tabla 1) se cataloga como una pancreatitis aguda inducida por hipertrigliceridemia y se estadifica con los siguientes scores: APACHE 15, mortalidad 30.4\%; MARSHALL: 1; BISAP: 1; RAMSAY: 5; RAMSON: 8; $y$, por estudio tomográfico Balthazar: B.

Tabla 1. Exámenes paraclínicos de ingreso

\begin{tabular}{|c|c|}
\hline Perfil hemático & $\begin{array}{l}\text { Leucocitos } 12.88 \times 103 / \mathrm{uL} \text {, granulocitos } 76 \% \text {, linfocitos } \\
16 \% \text {, hemoglobina } 28.1 \mathrm{~g} / \mathrm{dL} \text {, hematocrito } 32.4 \% \text {, } \\
\text { plaquetas } 361 \text { × } 103 / \mathrm{uL} \text {; se evidencia muestra lipémica. }\end{array}$ \\
\hline Perfil hepático & $\begin{array}{l}\text { AST } 60.0 \mathrm{U} / \mathrm{L} \text {, ALT } 45.0 \mathrm{U} / \mathrm{L} \text {, bilirrubina total } 19.3 \mathrm{mg} / \\
\mathrm{dL} \text {, bilirrubina directa } 15.6 \mathrm{mg} / \mathrm{dL} \text {, amilasa } 890 \mathrm{U} / \mathrm{L} \text {, } \\
\text { lipasa } 1080 \mathrm{U} / \mathrm{L} \text {. }\end{array}$ \\
\hline Perfil renal & Urea 18.6 mg/dL, creatinina 0.60 mg/dL. \\
\hline Marcadores de inflamación & PCR 198.1 mg/L, deshidrogenasa láctica 1560.0 U/L. \\
\hline Electrolitos & $\begin{array}{l}\text { Sodio } 142.7 \mathrm{mmol} / \mathrm{L} \text {, potasio } 5.4 \mathrm{mmol} / \mathrm{L} \text {, cloro } 116.9 \\
\mathrm{mmol} / \mathrm{L} \text {, calcio } 6.9 \mathrm{mmol} / \mathrm{L} \text {. }\end{array}$ \\
\hline Gasometría arterial & $\begin{array}{l}\text { pH 7.0, pCO2 11.7, pO2 154.5, HCO3 } 5.9 \mathrm{mmol} / \mathrm{L}, \mathrm{BE} \\
\text {-24.5, SatO2 97.7\% }\end{array}$ \\
\hline Perfil Lipídico & $\begin{array}{l}\text { Colesterol total } 1270.0 \mathrm{mg} / \mathrm{dL}, \mathrm{HDL} 285.0 \mathrm{mg} / \mathrm{dL} \text {, LDL } \\
431 \mathrm{mg} / \mathrm{dL} \text {, triglicéridos } 7080.0 \mathrm{mg} / \mathrm{dL} \text {. }\end{array}$ \\
\hline Ecografía de abdomen & $\begin{array}{l}\text { Hepatopatía grasa moderada, signos de pancreatitis } \\
\text { aguda, líquido peripancreático, esplenomegalia. }\end{array}$ \\
\hline TC de abdomen y pelvis simple & $\begin{array}{l}\text { Signos de pancreatitis aguda, esplenomegalia, } \\
\text { hepatopatía grasa, folículos en ovarios. }\end{array}$ \\
\hline
\end{tabular}

ALT: alanina aminotransferasa, AST: aspartato aminotransferasa, PCR: proteína C reactiva, TC: tomografía computarizada.

En el transcurso de las primeras horas de hospitalización, se evidencia aumento del dolor abdominal, de intensidad 10/10, palidez generalizada, frecuencia cardiaca de 140 lpm, con deterioro neurológico, y presencia de acidosis metabólica. La paciente es intubada y conectada a ventilación mecánica.

Se inicia hidratación amplia, infusión de insulina y analgesia, sin resultados evidentes. Se indica realización de plasmaféresis, como terapia coadyuvante. El procedimiento se realizó mediante un catéter vascular de alto flujo, durante 3 horas, en el cual se extrajeron 3600 $\mathrm{ml}$ de plasma de aspecto lechoso. La muestra de suero tomada posterior a la primera sesión (Figura 1) tuvo una concentración de triglicéridos de $7080 \mathrm{mg} / \mathrm{dl}$, logrando un descenso el 50\% del valor. El procedimiento se repitió a las 24 y 48 horas, con un recambio de $4000 \mathrm{ml}$ de plasma. El resultado final de la concentración de triglicéridos posterior a las 3 sesiones fue de 406 $\mathrm{mg} / \mathrm{dl}$ (tabla 2).

Tabla 2. Exámenes de laboratorio según la sesión de plasmaféresis.

\begin{tabular}{lccc}
\hline Sesión de plasmaféresis & Primera & Segunda & Tercera \\
\hline Colesterol total $(\mathrm{mg} / \mathrm{dL})$ & 620 & 569 & 217 \\
HDL $(\mathrm{mg} / \mathrm{dL})$ & 37.7 & & \\
LDL $(\mathrm{mg} / \mathrm{dL})$ & 26.0 & & \\
Triglicéridos $(\mathrm{mg} / \mathrm{dL})$ & 3040.0 & 1700.0 & 406.0 \\
Amilasa $(\mathrm{U} / \mathrm{l})$ & 577.0 & 101.0 & 54 \\
Lipasa $(\mathrm{U} / \mathrm{l})$ & 388 & 80 & 48 \\
\hline
\end{tabular}




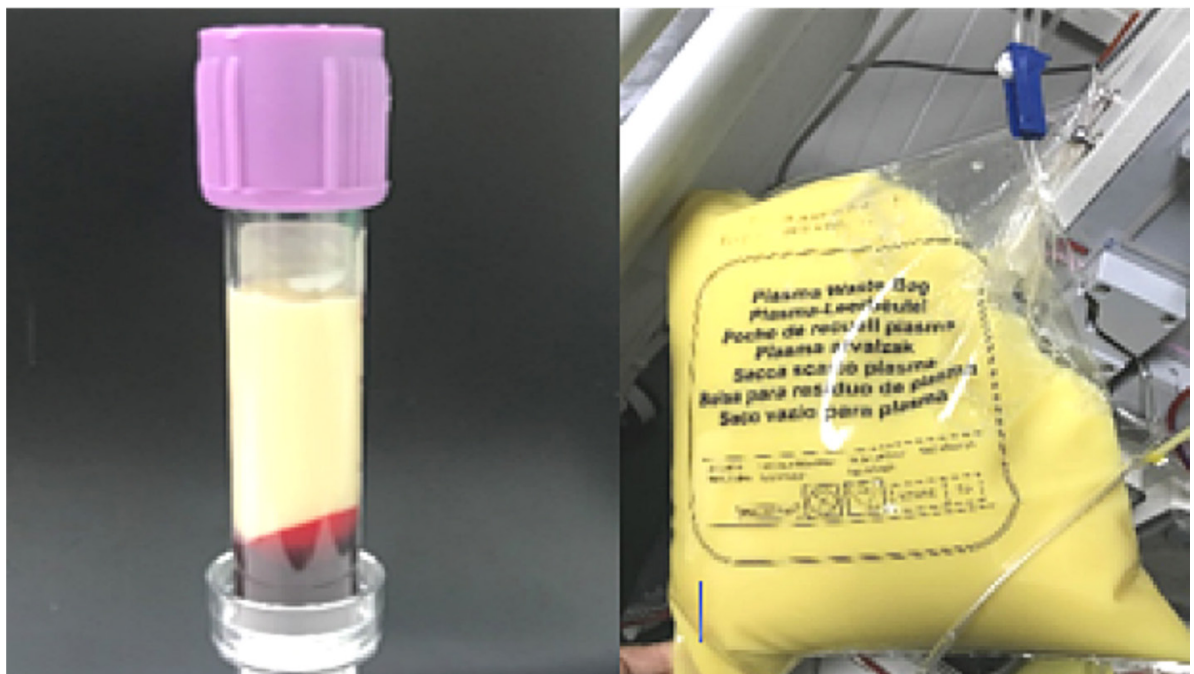

Figura 1. Muestra de plasma de aspecto lipídico.

A pesar que la plasmaféresis fue efectiva en esta paciente para la resolución del cuadro de pancreatitis, la evolución de su enfermedad a un shock séptico de foco de origen pulmonar por Acinetobacter Baumannii multirresitente, además del desarrollo de hemorragia intraencefálica, conllevó al fallecimiento de la paciente al noveno día de hospitalización.

\section{DISCUSIÓN}

La mortalidad en la pancreatitis secundaria a hipertrigliceridemia varía entre el $2 \%$ al $4 \%$ de los casos registrados en la literatura, superior a otras etiologías de pancreatitis aguda; los triglicéridos se pueden elevar en la pancreatitis leve o moderada de cualquier etiología, sin embargo, su elevación es de $<500 \mathrm{mg} / \mathrm{dl}$, no comparable con la pancreatitis por hipertrigliceridemia en el cual el nivel es $>1000 \mathrm{mg} / \mathrm{dl}$. La plasmaféresis ha demostrado una reducción de la mortalidad cuando se utiliza entre las primeras 24 a 48 horas, descendiendo los valores de triglicéridos en la primera sesión a niveles normales o cerca de rangos entre el 50 y $80 \%$ de los casos (11). De acuerdo a la Sociedad de Aféresis y la Asociación Americana de Medicina constituye una indicación III para el recambio plasmático terapéutico (11); se utilizó la terapia de plasmaféresis en el caso expuesto determinándose eficaz por el descenso de triglicéridos en el menor tiempo posible para corregir la cascada fisiopatológica. Los resultados obtenidos en el caso descrito con esta técnica fueron positivos y se determinó su efectividad por la evidente mejoría clínica y descenso en los niveles de triglicéridos. La escasa disponibilidad del recurso y los costos del mismo, hacen que muchas veces sea una terapia poco accesible.
En aquellos pacientes con hipertrigliceridemia grave la plasmaféresis constituye un tratamiento eficaz para disminuir el riesgo de desarrollar complicaciones y la mortalidad; se debe tener en cuenta las indicaciones del procedimiento, la duración y frecuencia con la cual se debe realizar la terapia, evaluando desde el ingreso la posibilidad de realizar la técnica sin demora y con ello disminuir la morbi-mortalidad de esta patología.

\section{AGRADECIMIENTOS}

Agradecemos al equipo que trabaja dentro de la Clínica Guayaquil y a todos aquellos que ayudaron a que esta publicación fuera posible.

\section{REFERENCIAS BIBLIOGRÁFICAS}

1. Joglekar K, Brannick B, Kadaria D, Sodhi A. Therapeutic plasmapheresis for hypertriglyceridemia-associated acute pancreatitis: case series and review of the literature. Ther Adv Endocrinol Metab. 2017;8(4): 59-65. doi: 10.1177/2042018817695449

2. Rincón Sánchez RAM, Montaño-Padilla GS, Concha Mejía A, Rodríguez Ruíz KD. Utilidad de la plasmaféresis en la pancreatitis aguda por hipertrigliceridemia: A propósito de un caso. Rev Colomb Gastroenterol. 2020;35(2 SE-Reporte de Casos): 226-31. doi: 10.22516/25007440.350

3. Herrera Del Águila DD, Garavito Rentería J, Linarez Medina K, Lizarzaburu Rodríguez V. Pancreatitis aguda por hipertrigliceridemia severa: reporte de caso y revisión de la literatura. Rev Gastroenterol Peru. 2015;35(2): 159-64

4. Garg R, Rustagi T. Management of Hypertriglyceridemia Induced Acute Pancreatitis. Biomed Res Int. 2018;2018: 4721357. doi: 10.1155/2018/4721357

5. Stefanutti C, Labbadia G, Morozzi C. Severe hypertriglyceridemia-related acute pancreatitis. 
Ther Apher Dial Off peer-reviewed J Int Soc Apher Japanese Soc Apher Japanese Soc Dial Ther. 2013;17(2): 130-7. doi: 10.1111/1744-9987.12008

6. Rawla P, Sunkara T, Thandra KC, Gaduputi V. Hypertriglyceridemia-induced pancreatitis: updated review of current treatment and preventive strategies. Clin J Gastroenterol. 2018;11(6): 441-8. doi: 10.1007/s12328-018-08811

7. Marín-Sánchez JA, Jiménez Sánchez HC, Ramírez Chaparro CM. Pancreatitis aguda severa por hipertrigliceridemia en el adulto: Presentación de caso clínico, diagnóstico y tratamiento. Rev Colomb Gastroenterol. 2018;33 (4 SE-Reporte de Casos): 459-63. doi: 10.22516/25007440.315

8. Senosiain Lalastra C, Tavío Hernández E, Moreira Vicente V, Maroto Castellanos M, García Sánchez MC, Aicart Ramos M, y col. Pancreatitis aguda por hipertrigliceridemia. Gastroenterol Hepatol. 2013;36(4): 274-9. doi: 10.1016/j. gastrohep.2012.11.006
9. Izquierdo-Ortiz MJ, Abaigar-Luquin $P$. Hipertrigliceridemia severa. Tratamiento con plasmaféresis. Nefrologia. 2012;32(3):417-8. doi: 10.3265/Nefrologia.pre2012.Feb.11394

10. Song X, Shi D, Cui Q, Yu S, Yang J, Song P, et al. Intensive insulin therapy versus plasmapheresis in the management of hypertriglyceridemiainduced acute pancreatitis (Bi-TPAl trial): study protocol for a randomized controlled trial. Trials. 2019;20(1):365. doi: 10.1186/s13063-019-3498-x

11. Montaño-Padilla GS, Concha-Mejía A, RincónSánchez RA, Rodríguez-Ruiz K. Utilidad de la plasmaféresis en la pancreatitis aguda por hipertrigliceridemia: a propósito de un caso. Rev Col Gastroenterol. 2020: 35(2): 226-231. doi: 10.22516/25007440.350

Conflicto de intereses. No se declara conflicto de intereses

Fuente de financiamiento. Ninguna declarada por los autores

\section{ACERCA DE LOS AUTORES}

1. Danny Fernando Silva Cevallos. Médico de Universidad de Guayaquil. Médico Posgradista de Medicina Interna Universidad de Guayaquil. Jefe del área de Emergencias / Hospitalización. Clínica Guayaquil, Guayaquil-Ecuador.

ORCID: 0000-0003-1014-7607

2. Mirella Barrera Rivera. Médico Posgradista de Medicina Interna. Clínica Guayaquil, GuayaquilEcuador.

\section{ORCID: 0000-0002-7766-410X}

3. Diana Stefania Evangelista Barragán. Médico Universidad de Guayaquil. Máster en Neuropsicología y Educación: Rama Investigación, Universidad de la Rioja. Diplomado en liderazgo y toma de decisiones Universidad Benito Juárez G. Posgradista de Cardiología Universidad de Especialidades Espíritu Santo. Coordinador de docencia del Hospital General Guasmo Sur. Clínica Guayaquil, Guayaquil-Ecuador.

\section{ORCID: 0000-0001-6542-9930}

4. César Enrique Arreaga Pérez. Médico Postgradista de cardiología, Clínica Guayaquil, Guayaquil-Ecuador.

ORCID: 0000-0002-5645-9721 